\title{
Rotary Systems Versus Manual K-File System in Primary Molar Root Canals - In Vitro Study
}

\author{
Abdul Karim Jalal Al Rwaily* \\ General dental practitioner, Turaif National Dispensary, Turaif, Saudi Arabia
}

Received: 阱 September 25, 2018; Published: 畊 October 03, 2018

*Corresponding author: Abdul Karim Jalal Al Rwaily, General dental practitioner, Turaif National Dispensary, Turaif, Saudi Arabia

\begin{abstract}
Aim: To evaluate and compare the cleaning ability between two, rotary shaper and manual K-files in primary molar root canals.

Materials and Methods: Twenty extracted maxillary and mandibular primary molars and $10 \mathrm{~mm}$ length were cleaned in water and stored in $0.5 \%$ sodium hypochlorite for 1 week. Caries excavation was done. Coronal access was made. After irrigation of the root canal with normal saline, a K-file with a compatible diameter was introduced into the root canal and the canal length was determined. A K-file size \#15 was introduced into the root canal and $1 \mathrm{ml}$ Indian ink was injected into the orifice. The roots were then randomly divided into 2 groups.

Results: Group-A (K-File) was compared at Coronal, Middle and Apical thirds using Pearson's Chi-Square test. Results showed that Group-A (K-File) performed better at the apical thirds, reporting complete removal in $83.3 \%$ of cases followed by coronal third and the least percentage of complete removal was displayed in the middle third with only $43.3 \%$ of cases reporting complete removal. Group-B (rotary) was compared at Coronal, Middle and Apical thirds using Pearson's Chi-Square test. Results showed that Group-B (rotary) performed better at the Coronal and middle thirds, reporting complete removal in $76.7 \%$ of cases and the least percentage of complete removal was displayed in the apical third with only $23.3 \%$ of cases reporting complete removal. Group A showed a better performance by exhibiting complete removal in $83 \%$ of the cases, while remaining comparison showed a better result with nearly $76.7 \%$ of the cases reporting complete removal, and remainder cases reporting $50 \%$ and more or lesser ink removal.
\end{abstract}

Conclusion: Rotary files can be an acceptable alternative in primary teeth root canal preparation, as instrumentation time is reduced, and the prepared root canal is more conical which favors better obturation. Clinically, reduced instrumentation time would significantly improve patient cooperation and lessen fatigue of the operator.

Keywords: Rotary System; K-File Coronal Apical; Middle Third; Pediatric Dentistry

\section{Introduction}

The premature loss of primary teeth may cause changes in the chronology and sequence of eruption of permanent teeth. Maintenance of primary teeth until physiological exfoliation contributes to mastication, phonation and aesthetics and prevents space loss in children. The primary objective of pulp therapy is to maintain the integrity and health of the teeth and their supporting tissues. The main aim of cleaning and shaping the root canal system is removing soft and hard tissue, which contains bacteria, making space for irritants to the apical third and medicaments, and subsequent obturating material in the radicular structure. The outcome of endodontic treatment in primary teeth is dependent on microbial reduction because of chemo mechanical preparation, removal of residual pulp tissue and debris, and maintenance of the original canal curvature during instrumentation. Stainless steel hand instruments being used for cleaning and shaping of root canals are not efficient enough in preparation of narrow and curved canals due to their inherent stiffness. Rotary instruments were introduced to pediatric endodontics by Barr et al. 2000. Recently, nickel-titanium rotary instruments have been developed, and are now widely used as an efficient technique. The designs and high flexibility of $\mathrm{Ni}-\mathrm{Ti}$ files allow instruments to closely follow the original root canal path, especially in curved canals and 
procedural errors such as ledges, over-instrumentation and apical transportation are greatly reduced as well with reduced operating time. Rotary instrumentation characteristics have become significantly relevant when dealing with endodontic preparation of primary teeth, since appointment length is a crucial factor for pediatric patient compliance and maintaining the original path of the root canal is essential to ensure the integrity of the germ of the permanent successor. Rotary files also improve patient cooperation by shortening treatment time for cleaning canals. The study is designed to evaluate and compare the cleaning ability between rotary instruments and conventional manual K-files instruments in the preparation of primary molar root canals.

\section{Materials and Methods}

\section{Type of Study and Source of Data}

The present in vitro study was conducted in the Department of Pediatric dentistry,

Turaif National Dispensary, Turaif, KSA. Twenty extracted primary maxillary and mandibular molars with at least two-thirds root remaining were collected and stored in formalin.

\section{Methodology}

Twenty extracted maxillary and mandibular primary molars with at least two thirds of intact root, and $10 \mathrm{~mm}$ length were cleaned in water and stored in $0.5 \%$ sodium hypochlorite for 1 week. Caries excavation was done. Coronal access was made. After irrigation of the root canal with normal saline, a K-file with a compatible diameter was introduced into the root canal and the canal length was determined at $1 \mathrm{~mm}$ from the apex or root bevel. A K-file size \#15 was introduced into the root canal and 1-2 mL Indian ink was injected into the orifice until the ink leaked from apical foramen.

a) Group A [20 Canals]: The root canals were instrumented manually with K-files. b) Group B [20 Canals]: were cleaned with the rotary system, using $21 \mathrm{~mm}$. The instruments were used to the full length of the root canal, as for the single-length technique. The instrumentation sequence was 10/.04, 15/.05, 20/.06 and 25/.06.

The teeth were cleared for cleaning efficacy analysis i.e. the teeth were placed separately in jars with a lid, containing $5 \%$ formic acid and $5 \%$ nitric acid for 3 days. The acid was renewed every 24 hours until the teeth were completely decalcified. The teeth were washed under running water for 8 hours and dehydrated in 70\% alcohol (for 16 hours, changed every hour), 90\% alcohol (for 3 hours, changed every hour) and 96\% alcohol (for 3 hours, changed every hour). After dehydration, the teeth were placed in methyl salicylate. At first the canals were separated from CEJ and were cut at $1 \mathrm{~mm}$ above the working length (2 $\mathrm{mm}$ upper than apex or root bevel) with Diamond disc, so that the apical section could be observed. Then the roots were cut from the mid part of the remaining canal (middle section). After clearing, each section was placed on a $1.5 \times 2$ inch red wax for easy observation. The removal of Indian ink from the cervical, middle, and apical thirds was analyzed with a stereoscopic with $\times 40$ of magnification and four-grade scoring system was used for grading the cleaning efficacy of the root canal system (RCS).

$$
\begin{aligned}
& 0=\text { total cleaning } \\
& 1=\text { more than } 50 \% \text { ink removal } \\
& 2=\text { less than } 50 \% \text { ink removal } \\
& 3=\text { no ink removal. }
\end{aligned}
$$

\section{Statistical Analysis}

The data obtained was subjected to Pearson's Chi-Square test depending on the normality of data and p-value $(<0.05)$ was

\begin{tabular}{|c|c|c|c|c|c|c|}
\hline \multicolumn{6}{|c|}{ Location Vs Score with Group-A } & \multirow{3}{*}{$P$ value } \\
\hline \multicolumn{6}{|c|}{ Cleaning Ability } & \\
\hline & & Total Ink Removal & $>50 \%$ Ink Removal & $<50 \%$ Ink Removal & No Ink Removal & \\
\hline & \multirow{2}{*}{ Coronal } & 18 & 5 & 7 & 0 & \multirow{6}{*}{0.004} \\
\hline \multirow{5}{*}{ Location } & & $60.00 \%$ & $16.60 \%$ & $23.30 \%$ & $0.00 \%$ & \\
\hline & \multirow{2}{*}{ middle } & 15 & 13 & 2 & 0 & \\
\hline & & $50.00 \%$ & $43.30 \%$ & $6.70 \%$ & $0.00 \%$ & \\
\hline & \multirow{2}{*}{ Apical } & 25 & 3 & 2 & 0 & \\
\hline & & $83.30 \%$ & $10.00 \%$ & $6.70 \%$ & $0.00 \%$ & \\
\hline
\end{tabular}
considered statistically significant.

\section{Results}

Table 1: Comparison of cleaning ability of Group-A (K-File) at Coronal, Middle and Apical thirds.

Table 1 showed that comparison of cleaning ability of Group-A (K-File) was compared at Coronal, Middle and Apical thirds using Pearson's Chi-Square test. Results showed that Group-A (K-File) performed better at the apical thirds, reporting complete removal in $83.3 \%$ of cases followed by coronal third and the least percentage of complete removal was displayed in the middle third with only 
$43.3 \%$ of cases reporting complete removal. There seemed a great difference in the cleaning ability of $\mathrm{k}$ file at different thirds of the canal. This association was statistically significant with a $p$ value of $<0.004$. Table 2 showed that Comparison of cleaning ability of Group-B was compared at Coronal, Middle and Apical thirds using Pearson's Chi-Square test. Results showed that Group-B performed better at the Coronal and middle thirds, reporting complete removal in $76.7 \%$ of cases and the least percentage of complete removal was displayed in the apical third with only $23.3 \%$ of cases reporting complete removal. There seemed a great difference in the cleaning ability of rotary system at different thirds of the canal. This association was statistically significant with a p value of $<0.001$.

Table 2: Comparison of cleaning ability of Group-B (rotary) at Coronal, Middle and Apical thirds.

\begin{tabular}{|c|c|c|c|c|c|c|}
\hline \multicolumn{6}{|c|}{ Cleaning Ability } & \multirow{2}{*}{$P$ value } \\
\hline & & Total Ink Removal & $>\mathbf{5 0 \%}$ Ink Removal & $<50 \%$ Ink Removal & No Ink Removal & \\
\hline & \multirow{2}{*}{ Coronal } & 23 & 6 & 1 & 0 & \multirow{6}{*}{0.001} \\
\hline \multirow{5}{*}{ Location } & & $76.70 \%$ & $20.00 \%$ & $3.30 \%$ & $0.00 \%$ & \\
\hline & \multirow{2}{*}{ middle } & 23 & 6 & 1 & 0 & \\
\hline & & $76.70 \%$ & $20.00 \%$ & $3.30 \%$ & $0.00 \%$ & \\
\hline & \multirow{2}{*}{ Apical } & 22 & 7 & 1 & 0 & \\
\hline & & $73.30 \%$ & $23.30 \%$ & $3.30 \%$ & $0.00 \%$ & \\
\hline
\end{tabular}

Table 3 describes the frequency distribution of scores obtained using three methods at the coronal third. We can clearly observe that Group A, showed complete removal in only $60 \%$ of the cases, while remaining cases reported $50 \%$ or more. Group B, the second method in comparison showed a better result with nearly $76.7 \%$ of the cases reporting complete removal, and remainder percentage reporting 50\% and more or lesser ink removal. There seemed to be a great difference in the performance of the cleaning methods at the coronal third, and this was found to be statistically significant, showing association of rotary methods with better cleaning ability, with a $\mathrm{p}$ value of $0.004(\mathrm{p}<0.05)$. Table 4 describes the frequency distribution of scores obtained using three methods at the Middle third. We can clearly observe that Group A, showed complete removal in only half the cases, while remaining cases reported $50 \%$ or more and lesser ink removal. Group B, the second method in comparison showed a better result with $73.3 \%$ of the cases reporting complete removal, and about $27 \%$ reporting $50 \%$ and more or lesser ink removal. Group C, the third group in comparison surpasses both the former groups in cleaning ability at the coronal third with nearly $86.7 \%$ showing complete removal of ink, while only a minor percentage reporting more than $50 \%$ removal. There seemed to be a great difference in the performance of the cleaning methods at the middle third, and this was found to be statistically significant, showing association of rotary methods with better cleaning ability, with a $\mathrm{p}$ value of $<0.003(\mathrm{p}<0.05)$.

Table 2: Inter-group comparison of cleaning ability of rotary and manual Instrument at Coronal thirds of primary molars.

\begin{tabular}{|c|c|c|c|c|c|c|}
\hline \multicolumn{6}{|c|}{ Cleaning Ability } & \multirow{2}{*}{ P Value } \\
\hline Location & & Total Ink Removal & $>\mathbf{5 0 \%}$ Ink Removal & $<50 \%$ Ink Removal & No Ink Removal & \\
\hline & \multirow{2}{*}{ Coronal } & 18 & 5 & 7 & 0 & \multirow{6}{*}{0.004} \\
\hline & & $60.00 \%$ & $16.60 \%$ & $23.30 \%$ & $0.00 \%$ & \\
\hline & \multirow{2}{*}{ middle } & 23 & 6 & 1 & 0 & \\
\hline & & $76.70 \%$ & $20.00 \%$ & $3.30 \%$ & $0.00 \%$ & \\
\hline & \multirow{2}{*}{ Apical } & 25 & 4 & 1 & 0 & \\
\hline & & $3.30 \%$ & $0.00 \%$ & $83.30 \%$ & $13.30 \%$ & \\
\hline
\end{tabular}

Table 4: Inter-group comparison of cleaning ability of rotary and manual Instrument at Middle thirds of primary molars.

\begin{tabular}{|c|c|c|c|c|c|c|}
\hline \multicolumn{6}{|c|}{ Cleaning Ability } & \multirow{2}{*}{$P$ value } \\
\hline & & Total Ink Removal & $>50 \%$ Ink Removal & $<50 \%$ Ink Removal & No Ink Removal & \\
\hline & \multirow{2}{*}{ Coronal } & 15 & 13 & 2 & 0 & \multirow{6}{*}{0.004} \\
\hline \multirow{5}{*}{ Location } & & $50.00 \%$ & $43.30 \%$ & $6.70 \%$ & $0.00 \%$ & \\
\hline & \multirow{2}{*}{ middle } & 22 & 7 & 1 & 0 & \\
\hline & & $73.30 \%$ & $23.30 \%$ & $3.30 \%$ & $0.00 \%$ & \\
\hline & \multirow{2}{*}{ Apical } & 26 & 3 & 1 & 0 & \\
\hline & & $86.70 \%$ & $10 \%$ & $3.30 \%$ & $0.00 \%$ & \\
\hline
\end{tabular}


Table 5 describes the frequency distribution of scores obtained using three methods at the apical third. We can clearly observe that Group A, showed a better performance by exhibiting complete removal in $83 \%$ of the cases, while remaining comparison showed a better result with nearly $76.7 \%$ of the cases reporting complete removal, and remainder cases reporting 50\% and more or lesser ink removal. There seemed to be a large difference in the performance of the cleaning methods at the apical third, however, this was NOT found to be statistically significant, showing no clear association of rotary or manual methods with better cleaning ability, with a $\mathrm{p}$ value of $0.258(\mathrm{p}>0.05)$.

Table 5: Inter-group comparison of cleaning ability of rotary and manual Instrument at Apical thirds of primary molars.

\begin{tabular}{|c|c|c|c|c|c|c|}
\hline \multicolumn{6}{|c|}{ Cleaning Ability } & \multirow{2}{*}{$P$ value } \\
\hline & & Total Ink Removal & $>50 \%$ Ink Removal & $<50 \%$ Ink Removal & No Ink Removal & \\
\hline & \multirow{2}{*}{ Coronal } & 25 & 3 & 2 & 0 & \multirow{6}{*}{0.004} \\
\hline \multirow{5}{*}{ Location } & & $83.30 \%$ & $10.00 \%$ & $6.70 \%$ & $0.00 \%$ & \\
\hline & \multirow{2}{*}{ middle } & 23 & 6 & 1 & 0 & \\
\hline & & $76.70 \%$ & $20.00 \%$ & $3.30 \%$ & $0.00 \%$ & \\
\hline & \multirow{2}{*}{ Apical } & 23 & 7 & 0 & 0 & \\
\hline & & $76.70 \%$ & $23.30 \%$ & $0.00 \%$ & $0.00 \%$ & \\
\hline
\end{tabular}

\section{Discussion}

Endodontic treatment has evolved and got revolutionized over the years. The modern endodontic specialty practice has little resemblance to the traditional endodontic practice. In pediatric dentistry, pulpectomy is the preferred treatment for infected pulp tissue [1]. Significant alterations and complexities in root canal morphology of primary teeth demands improvement in the instruments to prevent undesirable complications without creating any iatrogenic events such as instrument fracture, external transportation, ledge or perforation [2]. One of the most important concerns in pediatric dentistry is the loss of necrotic primary molars leading to space loss. Although the morphology of root canals in primary teeth renders endodontic treatment difficult, pulpectomies of primary teeth with severe pulpal involvement should be considered as the treatment of choice [3]. A practical pulpectomy technique for the primary teeth should include the following features like fast procedure with short treatment time and minimal number of appointments, effective debridement of the root canal without weakening the tooth structure or endangering the underlining permanent teeth with minimal procedural complications and maintaining tooth function until it is naturally exfoliated [4].

Schilder H (1974) emphasized that the root canal should present a flare shape from apical to coronal, preserving the apical foramen and not altering the original canal curvature5. Thorough knowledge of the anatomical configurations of the dental pulp, and the possible variations is critical for successful endodontics [5]. Uncertainty of the root canal morphology increases the risk of transportation, ledge formation and even perforation, and often results in failure of the procedure. Negotiation and thorough instrumentation of bizarre and tortuous canals encased in roots programmed for physiological resorption are the main challenges for pulpectomy
[6]. The goals of biomechanical preparation are to eliminate microorganisms from the root canal system, to remove the pulp tissue that supports the microbial growth and to avoid debris forcing beyond apical foramen that may sustain inflammation. Until 1960, root canal instruments were produced of carbon steel which are now replaced by stainless steel alloys. Stainless steel files have also been shown to create aberrations, probably because of the inherent stiffness of stainless steel, which was compounded by instrument design and canal shape [7]. Weine et al. (1975) reported that most instrumentation techniques with stainless steel instruments in curved canals results in apical transportation. This makes obtaining a successful apical seal more difficult [8].

A good review of the physical characteristics of nickel titanium, including explanations of the stronger, high temperature form (austentite), and the weaker, low temperature form (martensite). The use of slow-speed high-torque Ni-Ti rotary instrumentation had been accepted in the last decade by manufacturers, clinicians, and researchers, leading to many iatrogenic errors. Ideally it should now be changed to slow-speed low-torque or, preferably, right-torque motors, since each instrument has a specific ideal right torque. The values are usually low for the smaller and less tapered instruments, and high for the bigger and more tapered ones. A new endodontic motor (step-motor) with computer controlled electronics allows fine adjustments for different brands in order not to exceed their elastic limit. Inherent to the material used, stainless steel files have a high stiffness that increases with increasing instrument size and causes high lateral forces in curved canals. These restoring forces attempt to return the instrument to its original shape and act on the canal wall during preparation, influencing the amount of dentin removed. The forces are particularly influential at the junction of the instrument tip and its cutting edges. This instrument's rigidity is responsible for straightening and its consequences in the apical, middle and coronal thirds. The resulting transportation and canal 
aberrations (including ledges, zipping's and perforations) leave a significant portion of the canal wall instrumented, along with the creation of an irregular cross sectional shape that is harder to obturate. The answer to canal distortion was the introduction of a series of reamers and files made from nickel titanium (Ni-Ti) rather than stainless steel. The unique property of super elasticity may allow Ni-Ti files to be placed in curved canals with lesser lateral force being exerted the endodontic treatment in children may be challenging and time consuming, especially during root canal preparation, which is one of the most important stages of endodontic therapy. Considering that the rotary instruments provide similar root canal cleaning compared to manual instruments with a shorter instrumentation time, their utilization is well indicated in Pediatric dentistry, especially when treating children with behavior management disorders.

In primary teeth, the physiologic root resorption begins soon after root completion creating difficulty in accurate working length determination [9]. The effects of instrumentation, medication, and filling materials on the succedaneum's teeth are uncertain which dissuade some clinicians from performing pulpectomy. Also, potential behavioral problems add to the dentist's reluctance to perform pulpectomy in young children. Since most of the primary molar root canals are presented with a myriad of anatomic variations due to physiologic deposition of dentine islands, the impact of these design modifications on root canal preparation on primary teeth is little known. The ink penetration and clearing technique is useful for studying the cleaning ability of the instrumentation and the morphology of human teeth as it makes the teeth transparent so that the pulp cavity and root canal walls can be diagnosed14. In our study we used India ink injection method for an analysis of the cleaning efficacy and the teeth were cleaned as reported by Silva et al. [10]. Results of present study are in accordance with [11], where it was observed that of the three groups namely manual instruments (K-files) and two rotary systems (Mtwo and Pro Taper), cleaning capacity was better in the coronal and middle thirds of the canal than in the apical third. In a study done by [12] compared cleaning efficacy of manual instrumentation and two rotary systems namely Mtwo and Wave.

One in the preparation of primary molar root canals, Mtwo and Wave One systems showed significant difference in cleaning ability in the coronal and middle third of the primary molar root canal as compared to manual instrumentation ( $\mathrm{K}$ file). In the apical third of the root canal, $\mathrm{K}$ file performed better than other file systems, but the difference was not found to be statistically significant. Whereas in the present study it was seen that $\mathrm{k}$ files performed better at apical third, while Hero shaper performed better than k-file and Mtwo in coronal and middle third which is statistically significant. The ability of Mtwo rotary files to clean the root canals depends on the instrument's cross-sectional design and flute. The new Mtwo files have an S-shaped cross section and a noncutting tip. Thus, their positive rake angle with two noncutting edges is responsible for effective dentine cutting and achieving symmetrical root canals. The low fracture rate of Mtwo may be due to the increasing pitch length from tip to shaft, which reduces the tendency of the files to screw-in, thus minimizing the risk of instrument fracture. In addition, the electric torque-limited handpiece used with Mtwo direct helps reduce file fractures. However, according to Bürklein and Schäfer, both the Endo IT motor and Mtwo direct hand piece control motors are equally safe and suitable for preparing curved canals. Because of the design features of Mtwo instruments, file deformation may be significantly more frequent than with other instruments.

Thus, the working parts of Mtwo files should be carefully examined after every use. However, in study done by [13], it was reported that canals prepared with manual k-files and Mtwo rotary files, no significant difference was observed between the apical, middle and coronal thirds in the primary and permanent teeth. However, factors such as operator experience or the forces applied could affect the results of study. Katge F et al. [14] studied the effectiveness of cleaning efficacy of Mtwo files with $\mathrm{H}$-files and reported that there was no significant difference between the two instrumentation techniques, which is in accordance with Barr et al (2000) and Silva et al. (2004). Hedstrom files were recommended since they remove hard tissue only on withdrawal and penetrate readily with a minimum of resistance, which prevents pushing infected material through the apices [15]. A study done Arun Kumar S et al. [16] in which comparison was made between the root canals debridement capability of two different Ni-Ti rotary files namely Hyflex files and Hero shaper files. The results indicate none of the file system could remove the contrast medium completely. This finding is like the previous studies with another Ni-Ti rotary file system. In this study results showed both Hyflex and Hero shaper files shows clear or less debris and smear layer in coronal and middle third but unable to produce the much clear surface in apical third of canal surface which was in line with the present study. Instruments with positive cutting angles cut more efficiently and may have a superior cleaning ability compared with those with neutral or negative cutting angles. In this study variation of cleaning efficacy of both files in apical third might be due to the differences in surface conditioning and the design of the files.

File designs, rotational speed, different sequences of instrumentation and surface conditioning of the instruments are important factors for efficient instrumentation in root canals. The usage of a larger final file would be more liable to remove a greater portion of debris from apical thirds with the removal of dentin. Guobin Yang et al. [17] concluded that the canals prepared with Pro Taper instruments showed smaller amounts of debris and smear layer remaining in the apical region than Hero shaper files. In the present study it was observed that though Hero shaper files were higher in cleaning ability than rotary files at the apical region, the 
highest was done by $\mathrm{k}$ files. In the present study, stereomicroscopic evaluation revealed that the canals prepared using rotary files were cleaner than manual K-files. The difference in cleanliness may be due to the operator's tendency to place hand instruments more coronally, while the rotary preparation path is not affected by the operator. Studies found no significant difference in cleaning efficacy for Ni-Ti rotary and manual K-files in primary teeth. In the present study, we noticed shorter times with rotary files in comparison to manual $\mathrm{K}$-files, a finding consistent with many with previous studies, due to the shorter root length in primary teeth, lower cutting resistance of deciduous dentin and the need to use fewer instruments. This factor in pediatric patient allows fast, safe and quality procedures, thereby reducing the patient's and professional's fatigue. Rotary instrumentation in primary teeth has several disadvantages such as the higher cost of Ni-Ti instruments, endodontic hand piece and requires clinical experience to become efficient with the technique

\section{Conclusion}

Rotary files can be an acceptable alternative in primary teeth root canal preparation, as instrumentation time is reduced, and the prepared root canal is more conical which favors better obturation. Clinically, reduced instrumentation time would significantly improve patient cooperation and lessen fatigue of the operator.

\section{References}

1. Madan N, Rathnam A, Shigli AL, Indushekar KR (2011) K-file vs ProFiles in cleaning capacity and instrumentation time in primary molar root canals: An in vitro study. J Indian Soc Pedod Prev Dent 29(1): 2-6.

2. Guelzow A, Stamm O, Martus P, Kielbassa AM (2005) Comparative study of six Rotary nickel-titanium systems and hand instrumentation for root canal preparation. Int Endod J 38(10): 743-752.

3. Schafer, E, Erler M, Dammaschke T (2016) Comparative study on the shaping ability and cleaning efficiency of rotary Mtwo instruments. Part b. Cleaning effectiveness and shaping ability in severely curved root canals of extracted teeth. Int Endod J 39(3): 203-212.

4. Kuo CI(2006) Application of Ni-Ti rotary files for pulpectomy in primary molars. J Dent Sci 1(1): 10-15.
5. Estrela C, Bueno MR, Sousa Neto MD, Pécora JD (2008) Method for determination of root curvature radius using cone-beam computed tomography images. Braz Dent J 19(2): 114-118.

6. Ahmed H (2013) Anatomical challenges, electronic working length determination and current developments in root canal preparation of primary molar teeth. Int Endod J 46(11): 1011-1022.

7. Walton RE (1976) Histologic evaluation of different methods of enlarging the pulp canal space. J Endod 2(10): 304-311.

8. Luiten DJ, Morgan LA, Baumgartner JC, Marshall JG (1995) A comparison of four instrumentation techniques on apical canal transportation. J Endod 21(1): 26-32.

9. Chougule RB, Padmanabhan MY, Mandal MS (2012) A comparative evaluation of root canal length measurement techniques in primary teeth. Pediatr Dent 34(3): 53-56.

10. Silva LA, Leonardo MR, Nelson Filho, P, Tanomaru JM (2004) Comparison of rotary and manual instrumentation techniques on cleaning capacity and instrumentation time in deciduous molars. J Dent Child 71(1): 4547.

11. Azar, Safi, Nikaein (2012) Cleaning ability of primary root canals by Mtwo and Pro Taper rotary systems and manual instruments in primary teeth. Dent Res J 9(2): 146-151.

12. Pathak S (2016) Comparison between manual and rotary instrumentation. CHRISMED J Health Res 3(1): 60-64.

13. Azar MR, Mokhtare M (2011) Rotary Mtwo system versus manual K-file instruments: Efficacy in preparing primary and permanent molar root canals. Indian J Dent Res 22(2): 377-381.

14. Katge F (2016) Comparison between Rotary and Manual Instrumentation Techniques. Int J Clin Pediatr Dentistry 9(2): 124-127.

15. Arun Kumar S, Lanke RB (2015) An Invitro-Comparative Evaluation of Cleaning Efficacy Of Hyflex And Hero Shaper Rotary Niti File Systems Scanning Electron Microscope Study. Int J Recent Sci Res 6(9): 63206324.

16. Yang G, Hongkun Wu A, Zheng BY, Zhang CH, Bsne HL, et al. (2008) Scanning electron microscopic evaluation of debris and smear layer remaining following use of Pro Taper and Hero Shaper instruments in combination with $\mathrm{NaOCl}$ and EDTA irrigation. Oral Surg Oral Med Oral Pathol Oral Radiol Endod 106(4): 63-71.

17. Musale PK, Mujawar SA (2014) Evaluation of the efficacy of rotary versus hand files in root canal preparation of primary teeth in vitro using CBCT. Eur Arch Paediatr Dent 15(2): 113-120.

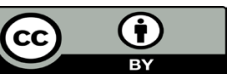

This work is licensed under Creative Commons Attribution 4.0 License

To Submit Your Article Click Here: Submit Article

DOI: 10.32474/IPDOAJ.2018.02.000132

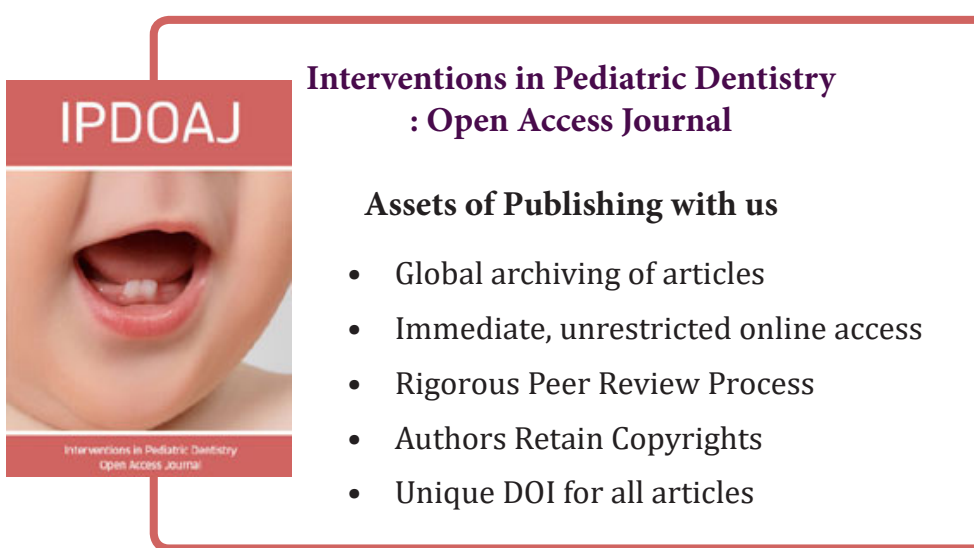

